\title{
Software Model Checking Improving Security of a Billion Computers
}

\author{
Patrice Godefroid
}

Microsoft Research 


\section{Acknowledgments}

- Joint work with Michael Levin (CSE) and others:

- Chris Marsh, Lei Fang, Stuart de Jong (CSE)

- interns Dennis Jeffries (06), David Molnar (07), Adam Kiezun (07), Bassem Elkarablieh (08), ...

- Thanks to the entire SAGE team and users!

- MSR: Ella Bounimova,...

- Z3: Nikolaj Bjorner, Leonardo de Moura,...

- WEX (Windows): Nick Bartmon, Eric Douglas,...

- Office: Tom Gallagher, Octavian Timofte,...

- SAGE users all across Microsoft! 


\section{References}

- see

- DART: Directed Automated Random Testing, with N. Klarlund and K. Sen, PLDI'2005

- Compositional Dynamic Test Generation, POPL'2007

- Automated Whitebox Fuzz Testing, with M. Levin and D. Molnar, NDSS'2008

- Demand-Driven Compositional Symbolic Execution, with S. Anand and N. Tillmann, TACAS'2008

- Grammar-Based Whitebox Fuzzing, with A. Kiezun and M. Levin, PLDI'2008

- Active Property Checking, with M. Levin and D. Molnar, EMSOFT'2008

- Precise Pointer Reasoning for Dynamic Test Generation, with B. Elkarablieh and M. Levin, ISSTA'2009 


\section{A Brief History of Software Model Checking}

- How to apply model checking to analyze software?

- "Real" programming languages (e.g., C, C++, Java),

- "Real" size (e.g., 100,000's lines of code).

- Two main approaches to software model checking:

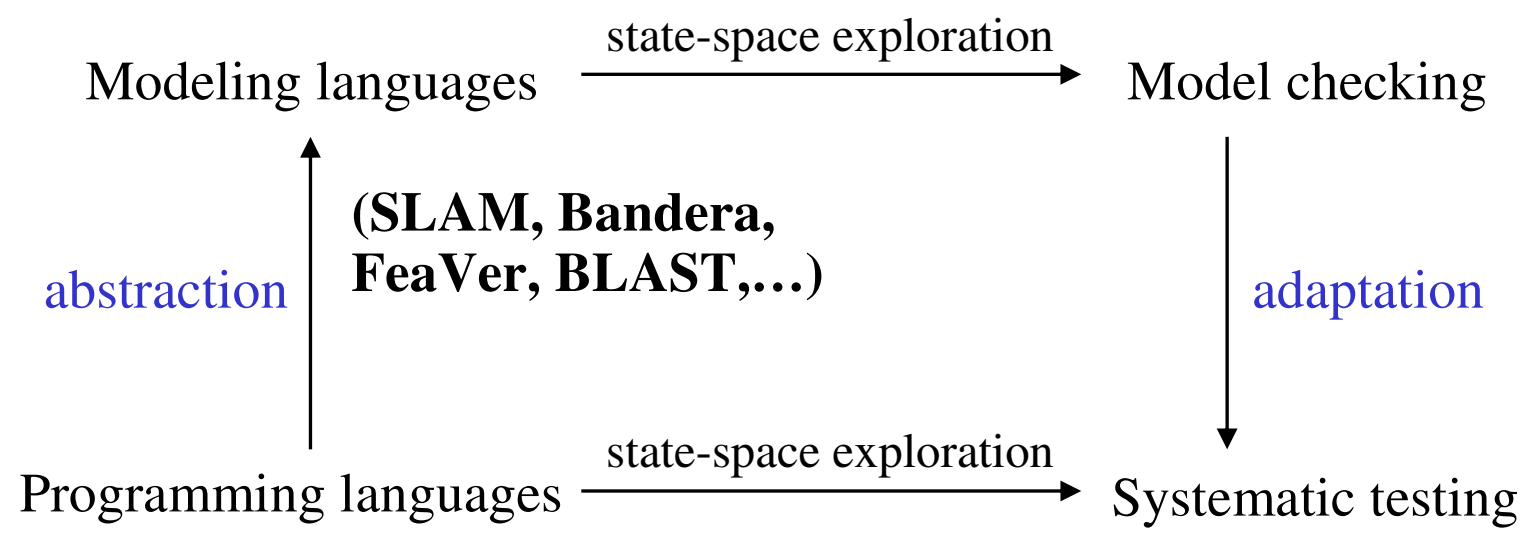

Concurrency: VeriSoft, JPF, CMC, Bogor, CHESS,...

Data inputs: DART, EXE, SAGE,...

Killer app: security biggest impact to date! 


\section{Security is Critical (to Microsoft)}

- Software security bugs can be very expensive:

- Cost of each Microsoft Security Bulletin: \$Millions

- Cost due to worms (Slammer, CodeRed, Blaster, etc.): \$Billions

- Most security exploits are initiated via files or packets

- Ex: Internet Explorer parses dozens of file formats

- Security testing: "hunting for million-dollar bugs"

- Write A/V (always exploitable), Read A/V (sometimes exploitable), NULL-pointer dereference, division-by-zero (harder to exploit but still DOS attacks), etc. 


\section{Hunting for Security Bugs}

- Main techniques used by "black hats":

- Code inspection (of binaries) and

- Blackbox fuzz testing

- Blackbox fuzz testing:

- A form of blackbox random testing [Miller+90]

- Randomly fuzz (=modify) a well-formed input

- Grammar-based fuzzing: rules that encode "well-formed"ness + heuristics about how to fuzz (e.g., using probabilistic weights)

- Heavily used in security testing

- Ex: July 2006 "Month of Browser Bugs"

- Simple yet effective: many bugs found this way...

- At Microsoft, fuzzing is mandated by the SDL

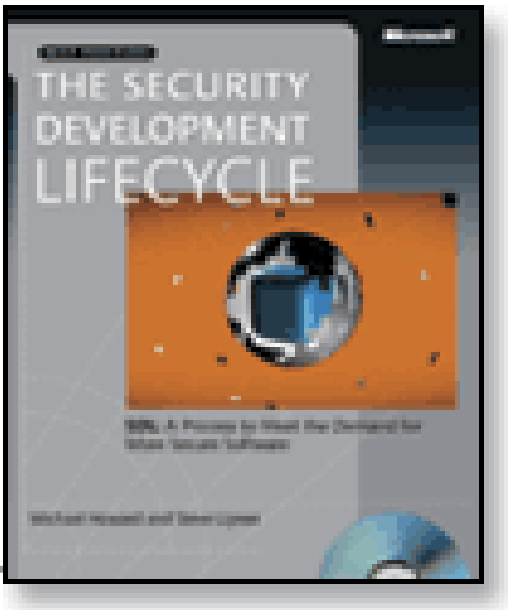




\section{Blackbox Fuzzing}

- Examples: Peach, Protos, Spike, Autodafe, etc.

- Why so many blackbox fuzzers?

- Because anyone can write (a simple) one in a week-end!

- Conceptually simple, yet effective...

- Sophistication is in the "add-on"

- Test harnesses (e.g., for packet fuzzing)

- Grammars (for specific input formats)

- Note: usually, no principled "spec-based" test generation

- No attempt to cover each state/rule in the grammar

- When probabilities, no global optimization (simply random walks) 


\section{Introducing Whitebox Fuzzing}

- Idea: mix fuzz testing with dynamic test generation

- Symbolic execution

- Collect constraints on inputs

- Negate those, solve with constraint solver, generate new inputs

- do "systematic dynamic test generation" (=DART)

- Whitebox Fuzzing = "DART meets Fuzz"

Two Parts:

1. Foundation: DART (Directed Automated Random Testing)

2. Key extensions ("Whitebox Fuzzing"), implemented in SAGE 


\section{Automatic Code-Driven Test Generation}

Problem:

Given a sequential program with a set of input parameters, generate a set of inputs that maximizes code coverage

= "automate test generation using program analysis"

This is not "model-based testing"

(= generate tests from an FSM spec) 


\section{How? (1) Static Test Generation}

- Static analysis to partition the program's input space $[$ King76,...]

- Ineffective whenever symbolic reasoning is not possible

- which is frequent in practice... (pointer manipulations, complex arithmetic, calls to complex OS or library functions, etc.)

Example:

int obscure(int $x$, int $y)\{$

if $(x==h a s h(y)) \operatorname{error}()$;

return 0 ;

\}
Can't statically generate values for $x$ and $y$ that satisfy " $x==$ hash $(y)$ " ! 


\section{How? (2) Dynamic Test Generation}

- Run the program (starting with some random inputs), gather constraints on inputs at conditional statements, use a constraint solver to generate new test inputs

- Repeat until a specific program statement is reached [Korel90,...]

- Or repeat to try to cover ALL feasible program paths: DART = Directed Automated Random Testing = systematic dynamic test generation [PLDI'05,...]

- detect crashes, assertion violations, use runtime checkers (Purify,...) 


\section{DART = Directed Automated Random Testing}

Example:

int obscure(int $x$, int $y)\{$

if $(x==h a s h(y)) \operatorname{error}()$;

return 0 ;

\}

- Observations:

- Dynamic test generation extends static test generation with additional runtime information: it is more powerful

- The number of program paths can be infinite: may not terminate!

- Still, DART works well for small programs (1,000s LOC)

- Significantly improves code coverage vs. random testing 


\section{DART Implementations}

- Defined by symbolic execution, constraint generation and solving

- Languages: C, Java, $x 86$, .NET,...

- Theories: linear arith., bit-vectors, arrays, uninterpreted functions,...

- Solvers: Ip_solve, CVCLite, STP, Disolver, Z3,...

- Examples of tools/systems implementing DART:

- EXE/EGT (Stanford): independent ['05-'06] closely related work

- CUTE = same as first DART implementation done at Bell Labs

- SAGE (CSE/MSR) for $\times 86$ binaries and merges it with "fuzz" testing for finding security bugs (more later)

- PEX (MSR) for NET binaries in conjunction with "parameterized-unit tests" for unit testing of .NET programs

- YOGI (MSR) for checking the feasibility of program paths generated statically using a SLAM-like tool

- Vigilante (MSR) for generating worm filters

- BitScope (CMU/Berkeley) for malware analysis

- CatchConv (Berkeley) focus on integer overflows

- Splat (UCLA) focus on fast detection of buffer overflows

- Apollo (MIT) for testing web applications ...and more! 


\section{DART Summary}

- DART attempts to exercise all paths (like model checking)

- Covering a single specific assertion (verification): hard problem (often intractable)

- Maximize path coverage while checking thousands of assertions all over: easier problem (optimization, best-effort, tractable)

- Better coverage than pure random testing (with directed search)

- DART can work around limitations of symbolic execution

- Symbolic execution is an adjunct to concrete execution

- Concrete values are used to simplify unmanageable symbolic expressions

- Randomization helps where automated reasoning is difficult

- Comparison with static analysis:

- No false alarms (more precise) but may not terminate (less coverage)

- "Dualizes" static analysis: static may vs. DART must

-Whenever symbolic exec is too hard, under-approx with concrete values

- If symbolic execution is perfect, no approx needed: both coincide! 


\section{Whitebox Fuzzing [NDSS'08]}

- Whitebox Fuzzing = "DART meets Fuzz"

- Apply DART to large applications (not unit)

- Start with a well-formed input (not random)

- Combine with a generational search (not DFS)

- Negate 1-by-1 each constraint in a path constraint

- Generate many children for each parent run

- Challenge all the layers of the application sooner

- Leverage expensive symbolic execution

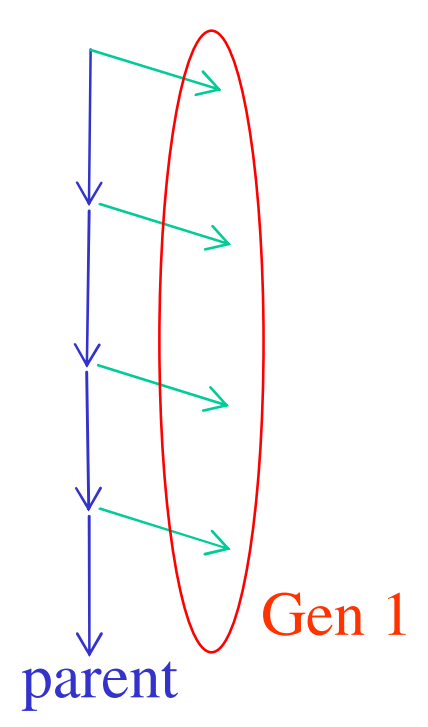

- Search spaces are huge, the search is partial... yet effective at finding bugs! 


\section{Example}

void top(char input[4])

\{

$$
\text { input }=\text { "good" }
$$

int cnt $=0$;

Path constraint:

if (input $[0]==$ ' $b$ ') cnt++; $I_{0} !={ }^{\prime} b$ '

if (input $[1]==$ ' $a$ ') cnt++; $I_{1} !={ }^{\prime} a$ '

if (input [2] == 'd') cnt++; $I_{2} !=$ 'd'

if (input [3] == '!') cnt++; $I_{3}$ !='!'

if $($ cnt $>=3) \operatorname{crash}()$;

\}

Negate each constraint in path constraint

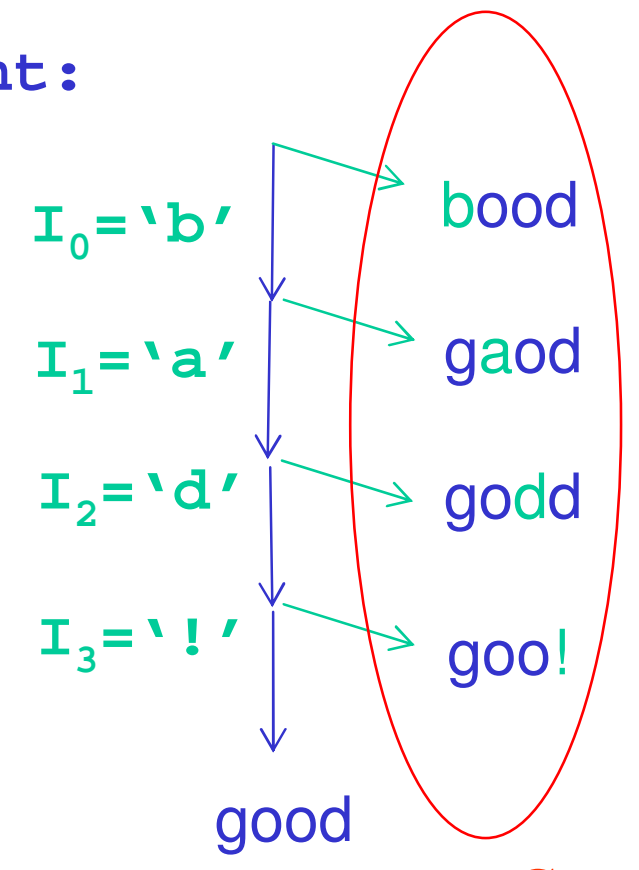

Gen 1 Solve new constraint new input 


\section{The Search Space}

void top(char input [4])

\{

int cnt $=0$;

if (input [0] == ' $b$ ') cnt++;

if (input $[1]==$ ' $a$ ') cnt++;

if (input [2] == 'd') cnt++;

if (input [3] == '!') cnt++;

if $(\mathrm{cnt}>=3) \operatorname{crash}()$;

\}

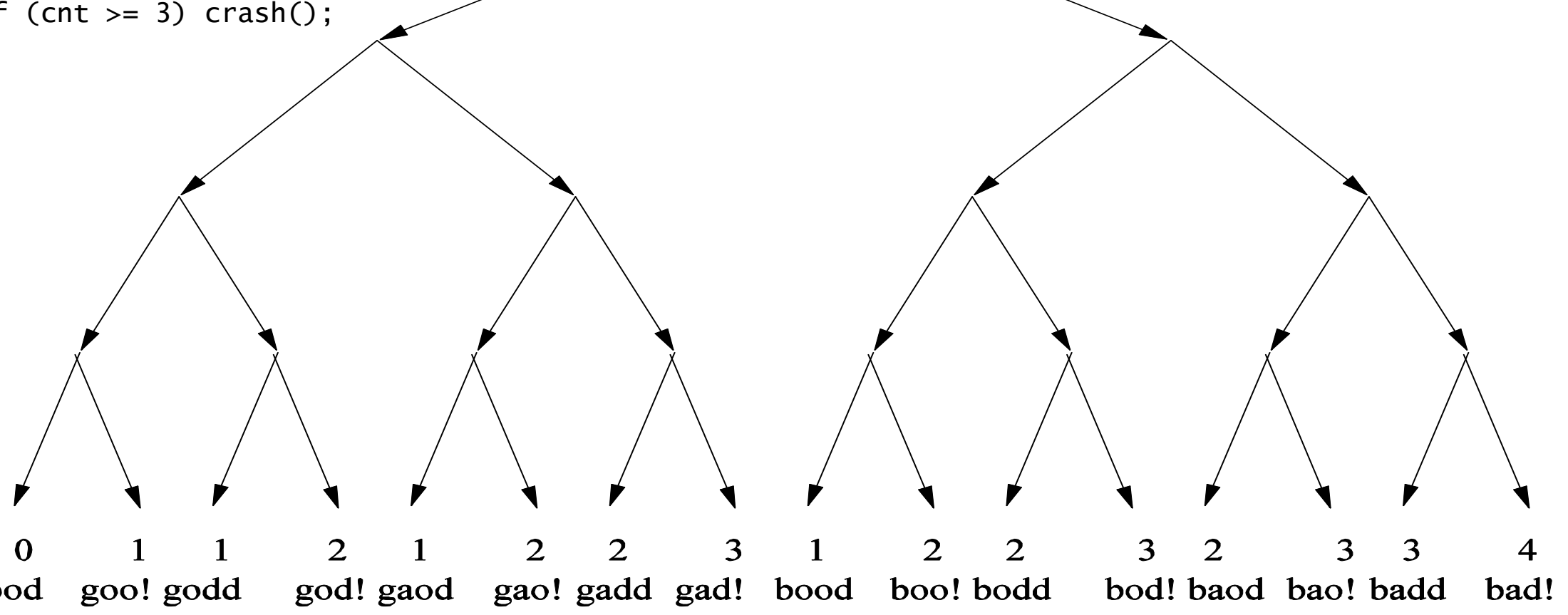




\section{SAGE (Scalable Automated Guided Execution)}

- Generational search introduced in SAGE

- Performs symbolic execution of $x 86$ execution traces

- Builds on Nirvana, iDNA and TruScan for x86 analysis

- Don't care about language or build process

- Easy to test new applications, no interference possible

- Can analyse any file-reading Windows applications

- Several optimizations to handle huge execution traces

- Constraint caching and common subexpression elimination

- Unrelated constraint optimization

- Constraint subsumption for constraints from input-bound loops

- "Flip-count" limit (to prevent endless loop expansions) 


\section{SAGE Architecture}

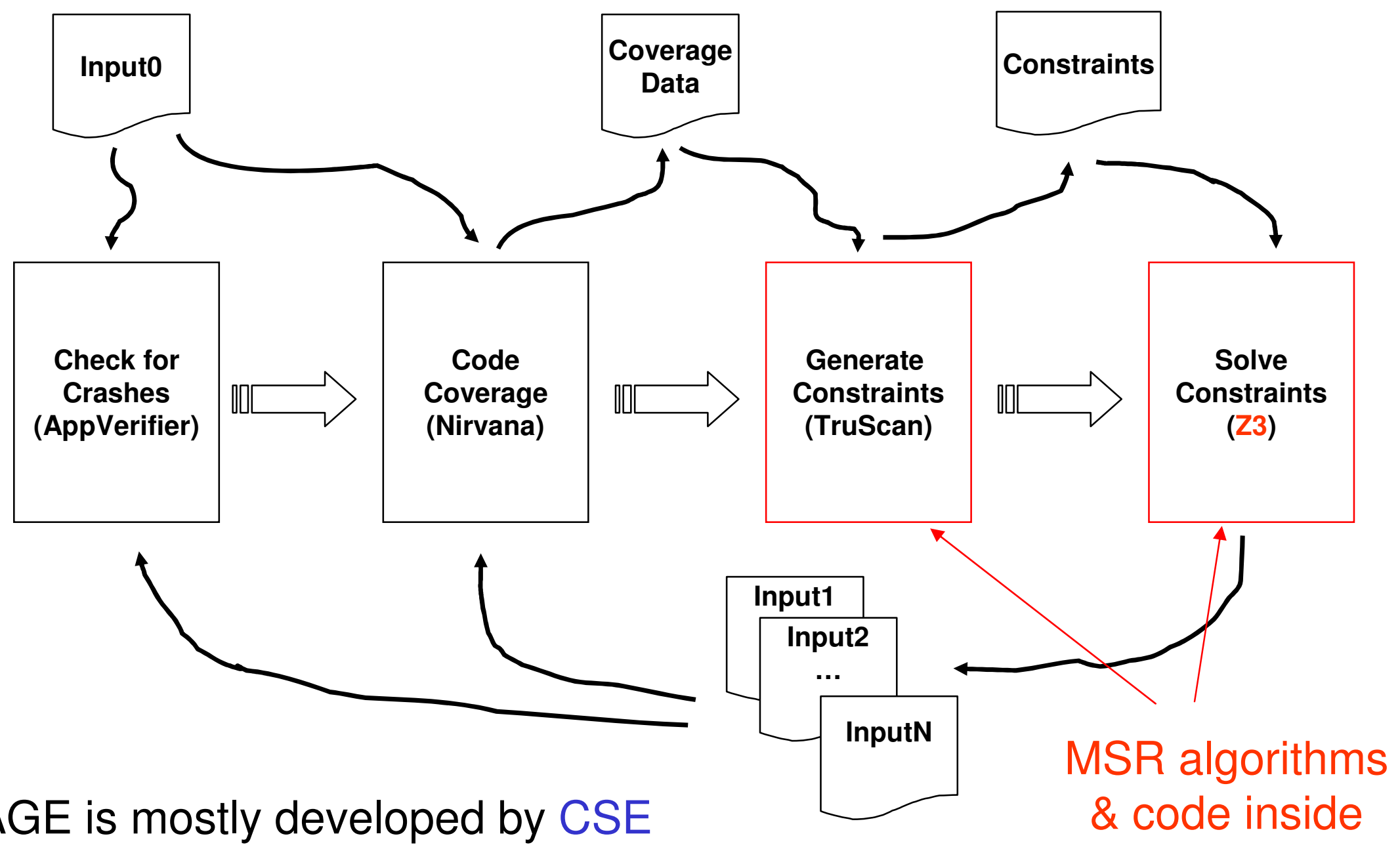




\section{Some Experiments Most much (100x) bigger than ever tried before!}

- Seven applications - 10 hours search each

App Tested \#Tests

ANI

Media1

Media2

Media3

Media4

Compressed

File Format

OfficeApp

3008

1527

\section{Mean Depth}

178

73

1100

608

883

65

6502
Mean \#Instr.

$2,066,087$

$3,409,376$

$271,432,489$

$54,644,652$

$133,685,240$

480,435

$923,731,248$
Mean Input

Size

5,400

65,536

27,335

30,833

22,209

634

45,064 


\section{Generational Search Leverages Symbolic Execution}

- Each symbolic execution is expensive

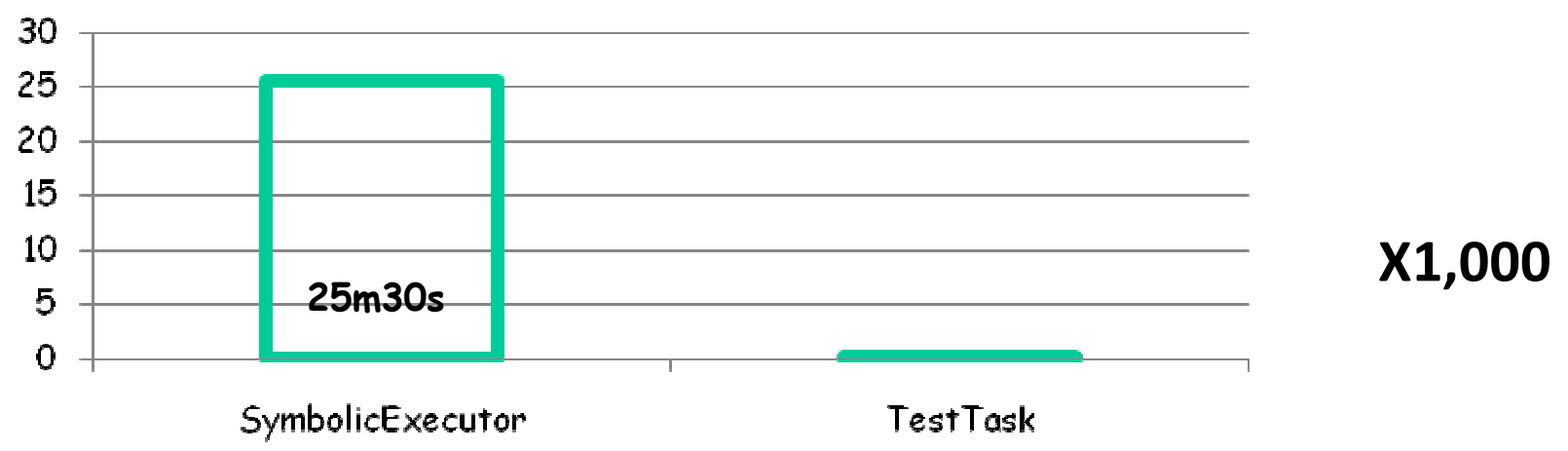

- Yet, symbolic execution does not dominate search time

10 hours

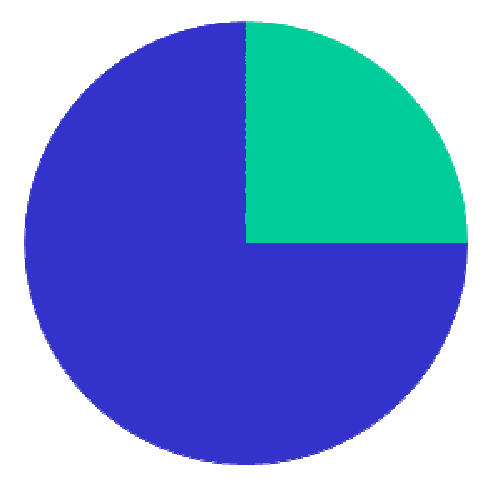

SymbolicExecutor

- Testing/Tracing/Coverage 


\section{SAGE Results}

Since April'07 $1^{\text {st }}$ release: many new security bugs found (missed by blackbox fuzzers, static analysis)

- Apps: image processors, media players, file decoders,...

- Bugs: Write A/Vs, Read A/Vs, Crashes,...

- Many triaged as "security critical, severity 1, priority 1" (would trigger Microsoft security bulletin if known outside MS)

- Example: WEX Security team for Win7

- Dedicated fuzzing lab with 100 s machines

- 100s apps (deployed on 1billion+ computers)

- $1 / 3$ of all fuzzing bugs found by SAGE!

- SAGE = gold medal at Fuzzing Olympics organized by SWI at BlueHat'08 (Oct'08)

- Credit due to entire SAGE team + users!

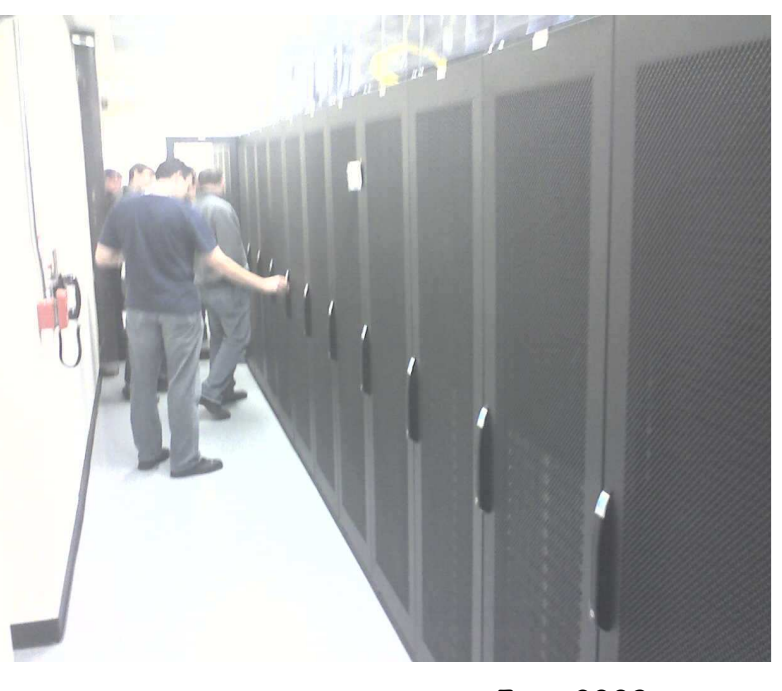




\section{WEX Fuzz Dashboard Snippet}

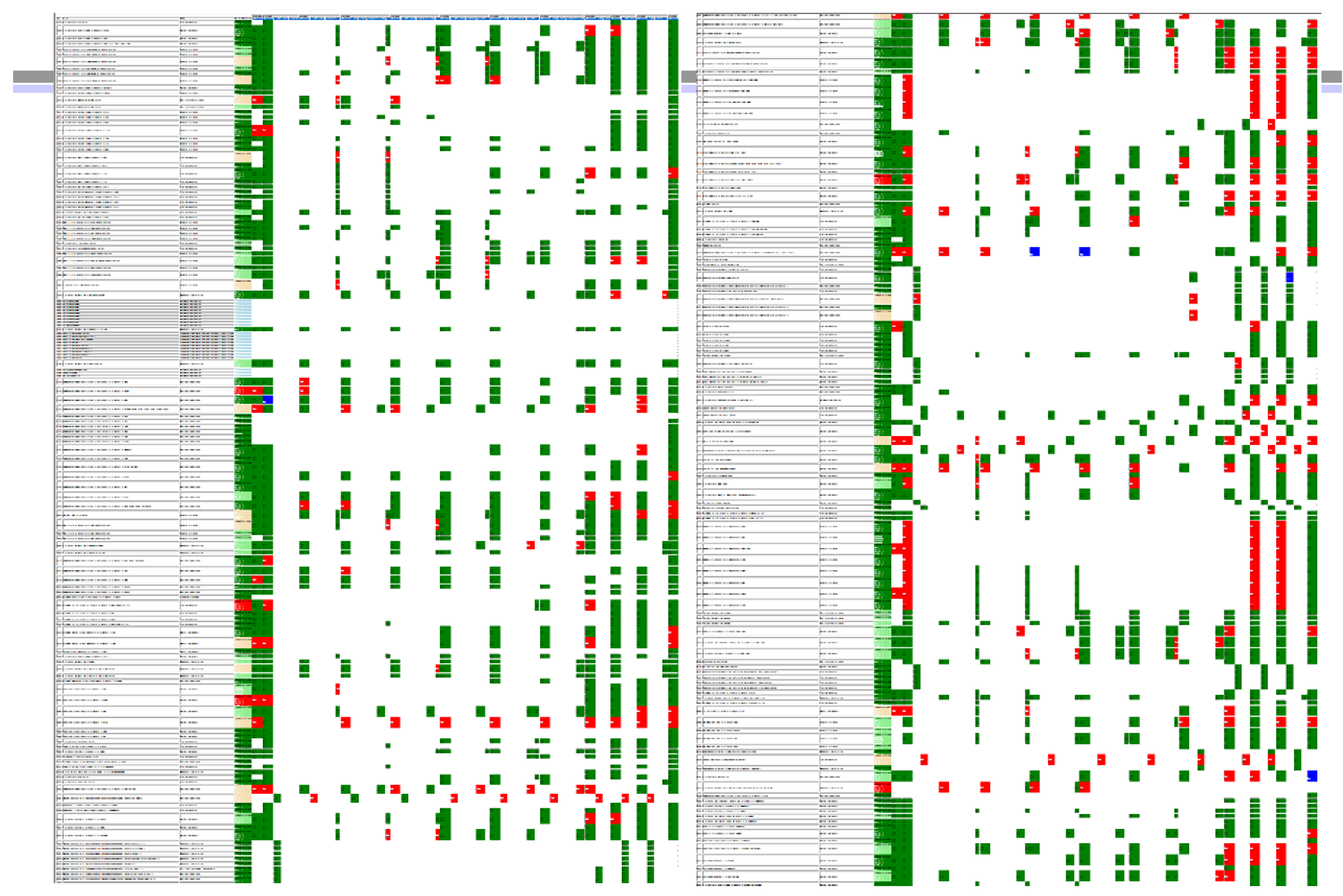




\section{WEX Fuzzing Lab Bug Yield for Win7}

How fuzzing bugs found (2006-2009) :

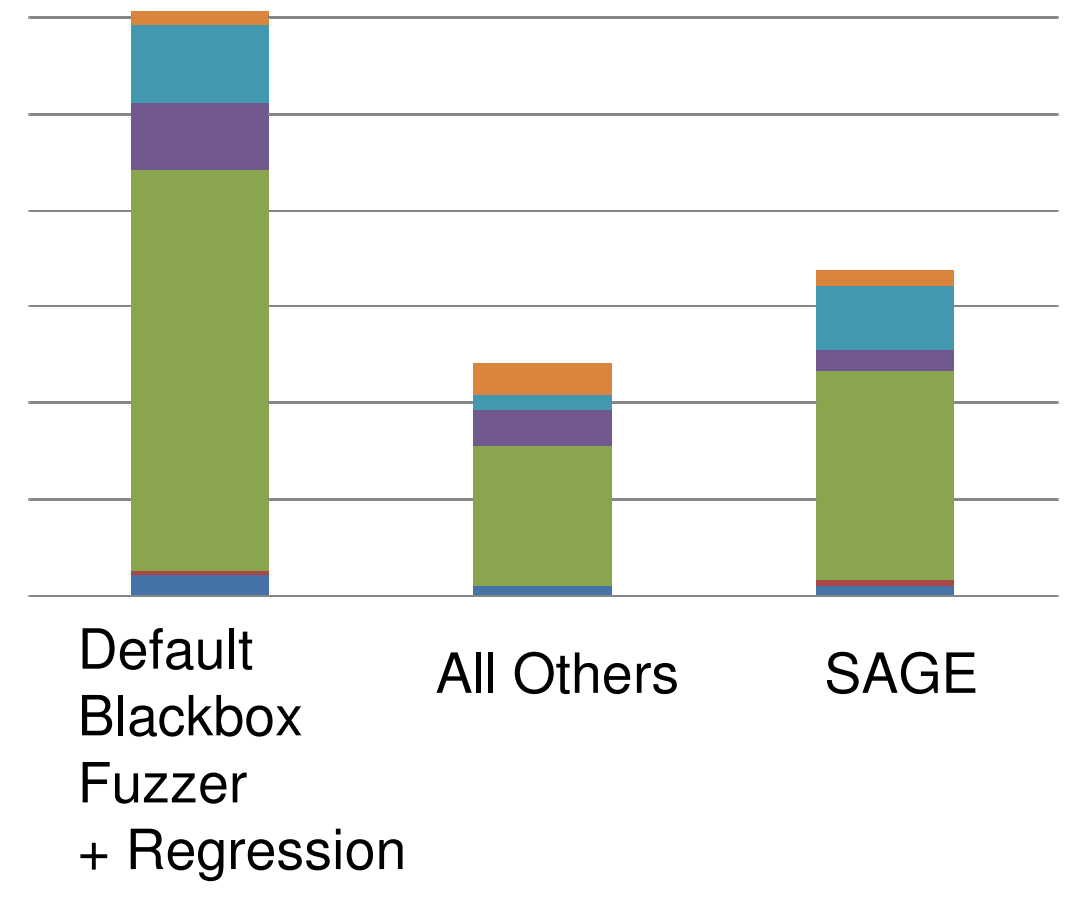

SAGE is running $24 / 7$ on 100 s machines: "the largest usage ever of any SMT solver" N. Bjorner + L. de Moura (MSR, Z3 authors)
- 100 s of apps, total number of fuzzing bugs is confidential

- But SAGE didn't exist in 2006

- Since 2007 (SAGE $1^{\text {st }}$ release), $\sim 1 / 3$ bugs found by SAGE

- But SAGE currently deployed on only $\sim 2 / 3$ of those apps

- Normalizing the data by $2 / 3$, SAGE found $1 / 2$ bugs

- SAGE is more CPU expensive, so it is run last in the lab, so all SAGE bugs were missed by everything else! 


\section{Zero to Crash in 10 Generations}

- Starting with 100 zero bytes ...

- SAGE generates a crashing test for Medial parser:

\begin{tabular}{|c|c|c|c|c|c|c|c|c|c|c|c|c|c|c|c|c|c|}
\hline $00000000 \mathrm{~h}:$ & 00 & 00 & 00 & 00 & 00 & 00 & 00 & 00 & 00 & 00 & 00 & 00 & 00 & 00 & 00 & 00 & \\
\hline 00000010h: & 00 & 00 & 00 & 00 & 00 & 00 & 00 & 00 & 00 & 00 & 00 & 00 & 00 & 00 & 00 & 00 & \\
\hline $00000020 \mathrm{~h}:$ & 00 & 00 & 00 & 00 & 00 & 00 & 00 & 00 & 00 & 00 & 00 & 00 & 00 & 00 & 00 & 00 & \\
\hline $00000030 h:$ & 00 & 00 & 00 & 00 & 00 & 00 & 00 & 00 & 00 & 00 & 00 & 00 & 00 & 00 & 00 & 00 & \\
\hline $00000040 \mathrm{~h}:$ & 00 & 00 & 00 & 00 & 00 & 00 & 00 & 00 & 00 & 00 & 00 & 00 & 00 & 00 & 00 & 00 & \\
\hline $00000050 \mathrm{~h}:$ & 00 & 00 & 00 & 00 & 00 & 00 & 00 & 00 & 00 & 00 & 00 & 00 & 00 & 00 & 00 & 00 & $\ldots \ldots \ldots \ldots$ \\
\hline $00000060 \mathrm{~h}:$ & 00 & 00 & 00 & 00 & & & & & & & & & & & & & \\
\hline
\end{tabular}

Generation 0 - seed file 


\section{Zero to Crash in 10 Generations}

- Starting with 100 zero bytes ...

- SAGE generates a crashing test for Medial parser:

\begin{tabular}{|c|c|c|c|c|c|c|c|c|c|c|c|c|c|c|c|c|c|}
\hline $00000000 \mathrm{~h}:$ & 52 & 49 & 46 & 46 & 00 & 00 & 00 & 00 & 00 & 00 & 00 & 00 & 00 & 00 & 00 & 00 & RIFE. \\
\hline $00000010 \mathrm{~h}:$ & 00 & 00 & 00 & 00 & 00 & 00 & 00 & 00 & 00 & 00 & 00 & 00 & 00 & 00 & 00 & 00 & 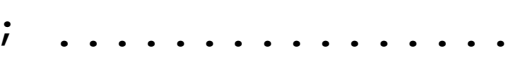 \\
\hline $00000020 h:$ & 00 & 00 & 00 & 00 & 00 & 00 & 00 & 00 & 00 & 00 & 00 & 00 & 00 & 00 & 00 & 00 & ; \\
\hline $00000030 \mathrm{~h}:$ & 00 & 00 & 00 & 00 & 00 & 00 & 00 & 00 & 00 & 00 & 00 & 00 & 00 & 00 & 00 & 00 & ; \\
\hline $00000040 h:$ & 00 & 00 & 00 & 00 & 00 & 00 & 00 & 00 & 00 & 00 & 00 & 00 & 00 & 00 & 00 & 00 & ; \\
\hline $00000050 \mathrm{~h}:$ & 00 & 00 & 00 & 00 & 00 & 00 & 00 & 00 & 00 & 00 & 00 & 00 & 00 & 00 & 00 & 00 & \\
\hline $00000060 \mathrm{~h}:$ & 00 & 00 & 00 & 00 & & & & & & & & & & & & & \\
\hline
\end{tabular}

Generation 1 


\section{Zero to Crash in 10 Generations}

- Starting with 100 zero bytes ...

- SAGE generates a crashing test for Medial parser:

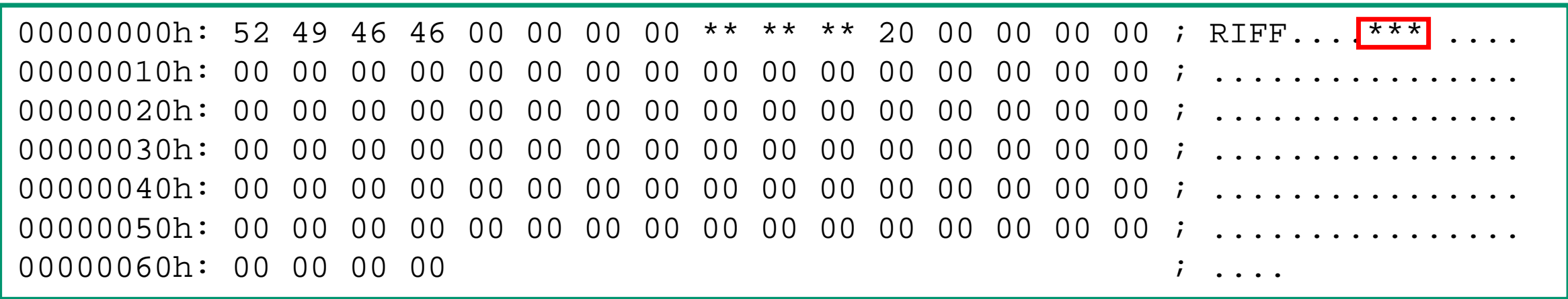

Generation 2 


\section{Zero to Crash in 10 Generations}

- Starting with 100 zero bytes ...

- SAGE generates a crashing test for Medial parser:

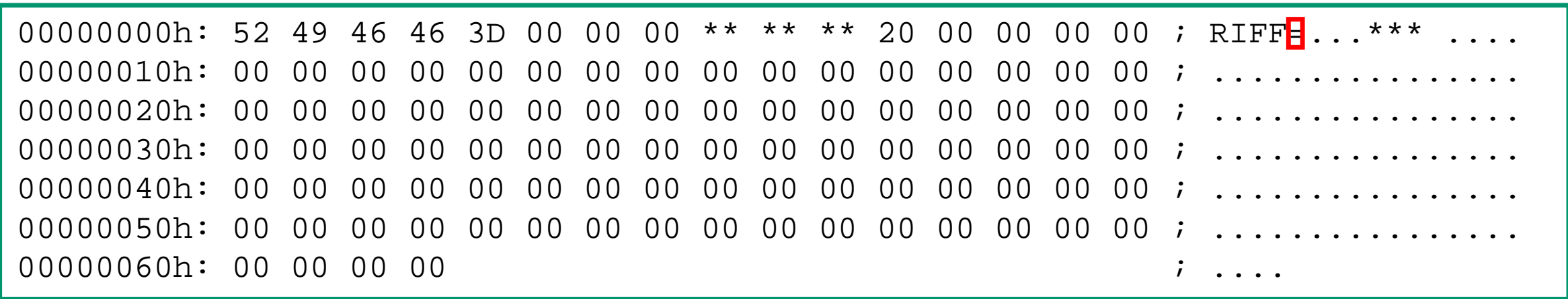

Generation 3 


\section{Zero to Crash in 10 Generations}

- Starting with 100 zero bytes ...

- SAGE generates a crashing test for Medial parser:

\begin{tabular}{|c|c|c|c|c|c|c|c|c|c|c|c|c|c|c|c|c|c|}
\hline 00000000h: & 52 & 49 & 46 & 46 & $3 \mathrm{D}$ & 00 & 00 & 00 & $\star \star$ & 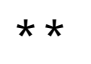 & $\star \star$ & 20 & 00 & 00 & 00 & 00 & $\cdot \star \star \star \quad \ldots$ \\
\hline $00000010 \mathrm{~h}:$ & 00 & 00 & 00 & 00 & 00 & 00 & 00 & 00 & 00 & 00 & 00 & 00 & 00 & 00 & 00 & 00 & ; \\
\hline $00000020 \mathrm{~h}:$ & 00 & 00 & 00 & 00 & 00 & 00 & 00 & 00 & 00 & 00 & 00 & 00 & 00 & 00 & 00 & 00 & ; \\
\hline $00000030 h:$ & 00 & 00 & 00 & 00 & 73 & 74 & 72 & 68 & 00 & 00 & 00 & 00 & 00 & 00 & 00 & 00 & trh \\
\hline $00000040 \mathrm{~h}:$ & 00 & 00 & 00 & 00 & 00 & 00 & 00 & 00 & 00 & 00 & 00 & 00 & 00 & 00 & 00 & 00 & ; \\
\hline $00000050 \mathrm{~h}:$ & 00 & 00 & 00 & 00 & 00 & 00 & 00 & 00 & 00 & 00 & 00 & 00 & 00 & 00 & 00 & 00 & . \\
\hline $00000060 \mathrm{~h}:$ & 00 & 00 & 00 & 00 & & & & & & & & & & & & & \\
\hline
\end{tabular}

Generation 4 


\section{Zero to Crash in 10 Generations}

- Starting with 100 zero bytes ...

- SAGE generates a crashing test for Medial parser:

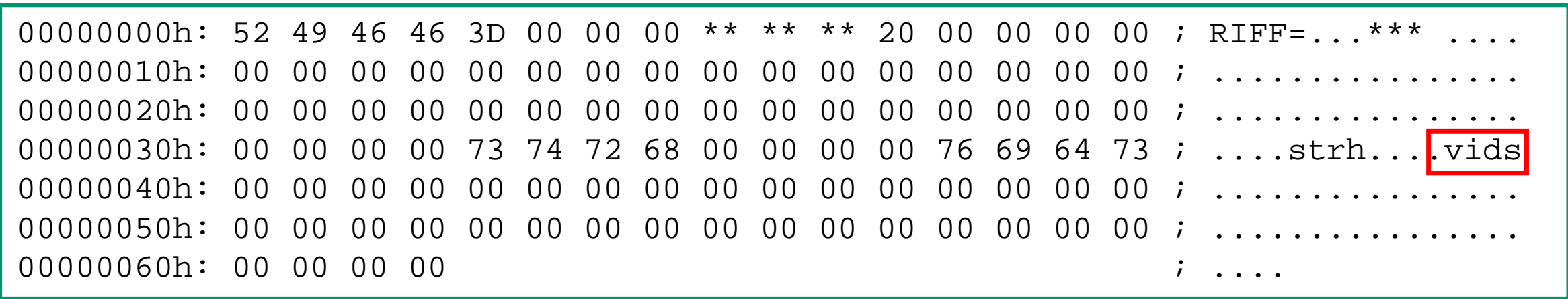

Generation 5 


\section{Zero to Crash in 10 Generations}

- Starting with 100 zero bytes ...

- SAGE generates a crashing test for Medial parser:

\begin{tabular}{|c|c|c|c|c|c|c|c|c|c|c|c|c|c|c|c|c|c|}
\hline $00000000 \mathrm{~h}:$ & 52 & 49 & 46 & 46 & $3 \mathrm{D}$ & 00 & 00 & 00 & $\star \star$ & 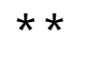 & $\star \star$ & 20 & 00 & 00 & 00 & 00 & 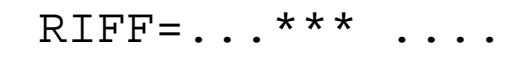 \\
\hline $00000010 \mathrm{~h}:$ & 00 & 00 & 00 & 00 & 00 & 00 & 00 & 00 & 00 & 00 & 00 & 00 & 00 & 00 & 00 & 00 & \\
\hline $00000020 \mathrm{~h}:$ & 00 & 00 & 00 & 00 & 00 & 00 & 00 & 00 & 00 & 00 & 00 & 00 & 00 & 00 & 00 & 00 & \\
\hline $00000030 \mathrm{~h}:$ & 00 & 00 & 00 & 00 & 73 & 74 & 72 & 68 & 00 & 00 & 00 & 00 & 76 & 69 & 64 & 73 & strh....vids \\
\hline $00000040 \mathrm{~h}:$ & 00 & 00 & 00 & 00 & 73 & 74 & 72 & 66 & 00 & 00 & 00 & 00 & 00 & 00 & 00 & 00 & strf $\ldots \ldots \ldots$ \\
\hline $00000050 \mathrm{~h}:$ & 00 & 00 & 00 & 00 & 00 & 00 & 00 & 00 & 00 & 00 & 00 & 00 & 00 & 00 & 00 & 00 & \\
\hline $00000060 \mathrm{~h}:$ & 00 & 00 & 00 & 00 & & & & & & & & & & & & & \\
\hline
\end{tabular}

Generation 6 


\section{Zero to Crash in 10 Generations}

- Starting with 100 zero bytes ...

- SAGE generates a crashing test for Medial parser:

\begin{tabular}{|c|c|c|c|c|c|c|c|c|c|c|c|c|c|c|c|c|c|}
\hline $00000000 \mathrm{~h}:$ & 52 & 49 & 46 & 46 & $3 \mathrm{D}$ & 00 & 00 & 00 & $\star \star$ & $\star \star$ & $\star \star$ & 20 & 00 & 00 & 00 & 00 & $\operatorname{RIFF}=\ldots \star \star \star \star$ \\
\hline $00000010 \mathrm{~h}:$ & 00 & 00 & 00 & 00 & 00 & 00 & 00 & 00 & 00 & 00 & 00 & 00 & 00 & 00 & 00 & 00 & \\
\hline $00000020 \mathrm{~h}:$ & 00 & 00 & 00 & 00 & 00 & 00 & 00 & 00 & 00 & 00 & 00 & 00 & 00 & 00 & 00 & 00 & ( \\
\hline $00000030 h:$ & 00 & 00 & 00 & 00 & 73 & 74 & 72 & 68 & 00 & 00 & 00 & 00 & 76 & 69 & 64 & 73 & ...strh.. \\
\hline $00000040 \mathrm{~h}:$ & 00 & 00 & 00 & 00 & 73 & 74 & 72 & 66 & 00 & 00 & 00 & 00 & 28 & 00 & 00 & 00 & ...strf.. \\
\hline $00000050 \mathrm{~h}:$ & 00 & 00 & 00 & 00 & 00 & 00 & 00 & 00 & 00 & 00 & 00 & 00 & 00 & 00 & 00 & 00 & \\
\hline $00000060 \mathrm{~h}:$ & 00 & 00 & 00 & 00 & & & & & & & & & & & & & \\
\hline
\end{tabular}

Generation 7 


\section{Zero to Crash in 10 Generations}

- Starting with 100 zero bytes ...

- SAGE generates a crashing test for Medial parser:

\begin{tabular}{|c|c|c|c|c|c|c|c|c|c|c|c|c|c|c|c|c|c|}
\hline $00000000 \mathrm{~h}:$ & 52 & 49 & 46 & 46 & 3D & 00 & 00 & 00 & $\star \star$ & $\star \star$ & $\star \star$ & 20 & 00 & 00 & 00 & 00 & $\operatorname{RIFF}=\ldots \star \star \star$ \\
\hline $00000010 \mathrm{~h}:$ & 00 & 00 & 00 & 00 & 00 & 00 & 00 & 00 & 00 & 00 & 00 & 00 & 00 & 00 & 00 & 00 & ; \\
\hline $00000020 \mathrm{~h}:$ & 00 & 00 & 00 & 00 & 00 & 00 & 00 & 00 & 00 & 00 & 00 & 00 & 00 & 00 & 00 & 00 & ; \\
\hline $00000030 h:$ & 00 & 00 & 00 & 00 & 73 & 74 & 72 & 68 & 00 & 00 & 00 & 00 & 76 & 69 & 64 & 73 & ....strh...vids \\
\hline $00000040 \mathrm{~h}:$ & 00 & 00 & 00 & 00 & 73 & 74 & 72 & 66 & 00 & 00 & 00 & 00 & 28 & 00 & 00 & 00 & ....strf....... \\
\hline $00000050 \mathrm{~h}:$ & 00 & 00 & 00 & 00 & 00 & 00 & 00 & 00 & 00 & 00 & 00 & 00 & C9 & $9 D$ & E4 & $4 \mathrm{E}$ & . . . . . E E \\
\hline $00000060 \mathrm{~h}:$ & 00 & 00 & 00 & 00 & & & & & & & & & & & & & -. \\
\hline
\end{tabular}

Generation 8 


\section{Zero to Crash in 10 Generations}

- Starting with 100 zero bytes ...

- SAGE generates a crashing test for Medial parser:

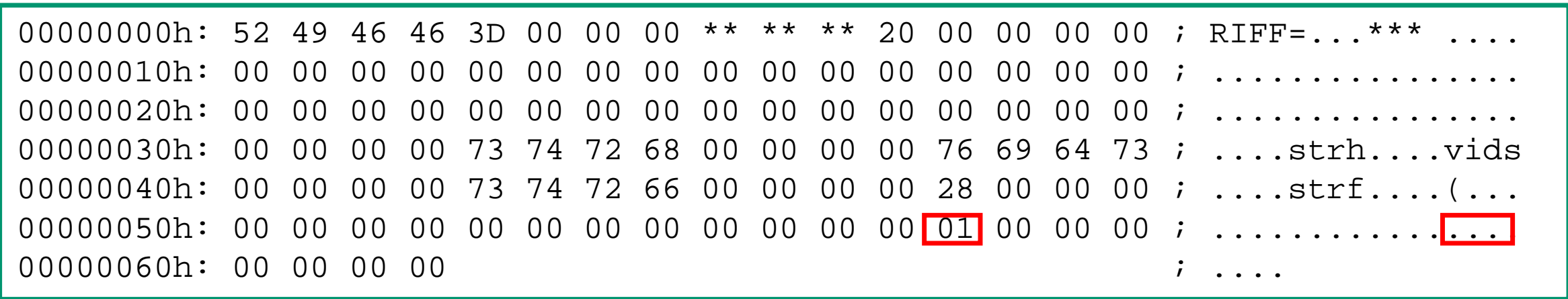

Generation 9 


\section{Zero to Crash in 10 Generations}

- Starting with 100 zero bytes ...

- SAGE generates a crashing test for Medial parser:

\begin{tabular}{|c|c|c|c|c|c|c|c|c|c|c|c|c|c|c|c|c|c|}
\hline $00000000 \mathrm{~h}:$ & 52 & 49 & 46 & 46 & 3D & 00 & 00 & 00 & ** & ** & 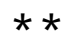 & 20 & 00 & 00 & 00 & 00 & $\operatorname{RIFF}=\ldots \star \star \star$ \\
\hline $00000010 \mathrm{~h}:$ & 00 & 00 & 00 & 00 & 00 & 00 & 00 & 00 & 00 & 00 & 00 & 00 & 00 & 00 & 00 & 00 & \\
\hline $00000020 \mathrm{~h}:$ & 00 & 00 & 00 & 00 & 00 & 00 & 00 & 00 & 00 & 00 & 00 & 00 & 00 & 00 & 00 & 00 & ( \\
\hline $00000030 \mathrm{~h}:$ & 00 & 00 & 00 & 00 & 73 & 74 & 72 & 68 & 00 & 00 & 00 & 00 & 76 & 69 & 64 & 73 & ....strh...vids \\
\hline $00000040 \mathrm{~h}:$ & 00 & 00 & 00 & 00 & 73 & 74 & 72 & 66 & B2 & 75 & 76 & $3 \mathrm{~A}$ & 28 & 00 & 00 & 00 & $=r f^{2} u v:(\ldots$ \\
\hline $00000050 \mathrm{~h}:$ & 00 & 00 & 00 & 00 & 00 & 00 & 00 & 00 & 00 & 00 & 00 & 00 & 01 & 00 & 00 & 00 & \\
\hline $00000060 \mathrm{~h}:$ & 00 & 00 & 00 & 00 & & & & & & & & & & & & & \\
\hline
\end{tabular}

Generation 10 - crash bucket 1212954973 !

Found after only 3 generations starting from seed 3 file on next slide 


\section{Different Seed Files, Different Crashes}

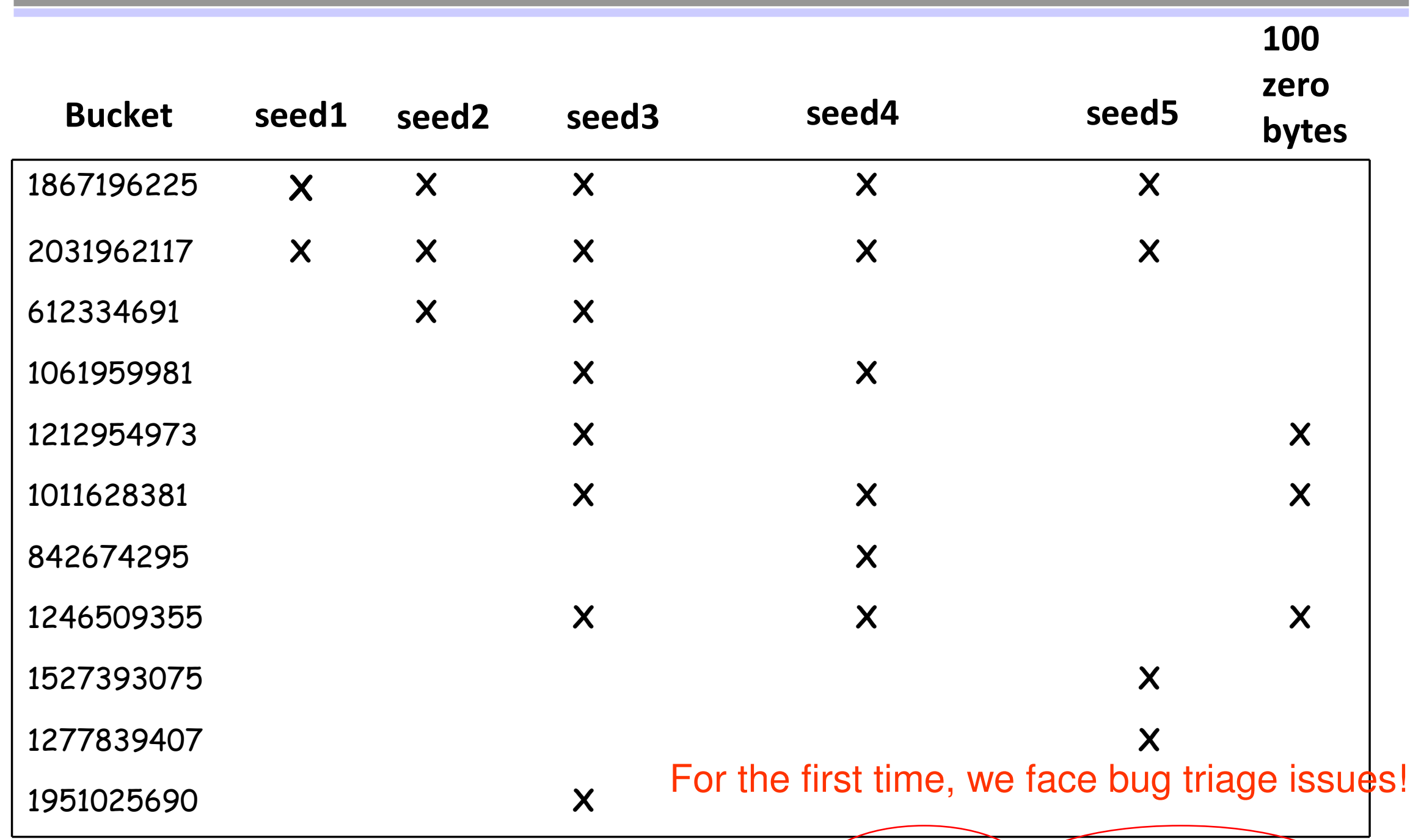

Media1: 60 machine-hours, 44598 total tests, 357 crashes, 12 unique buckets 


\section{Most Bugs Found are "Shallow"}

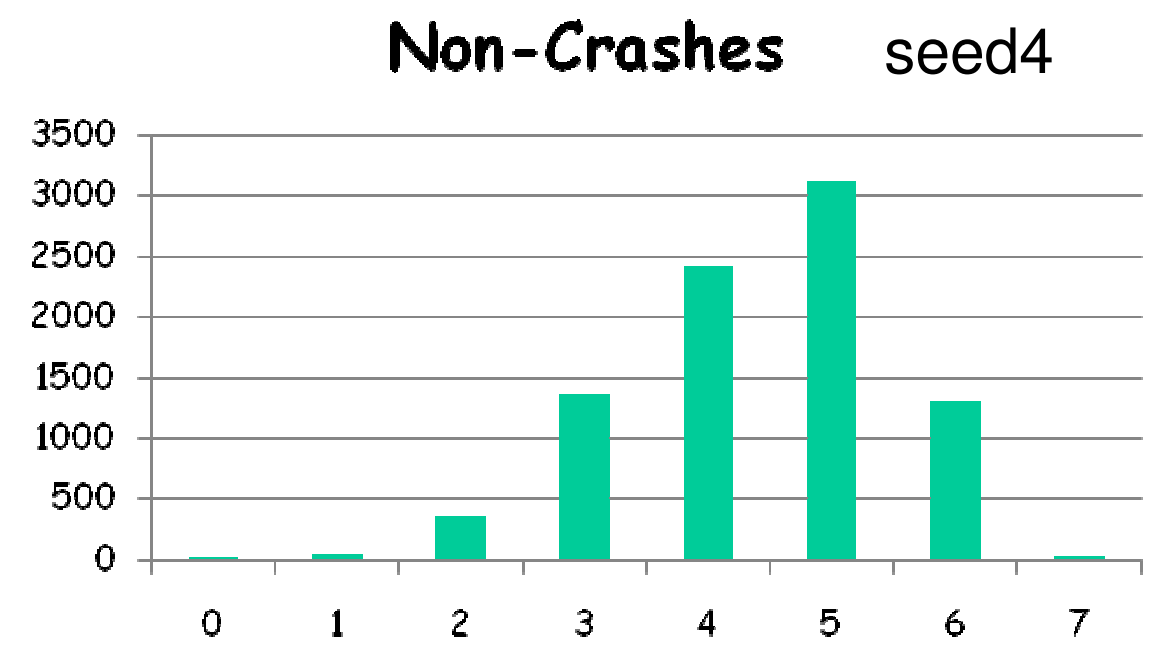

Crashes by Generation seed 4

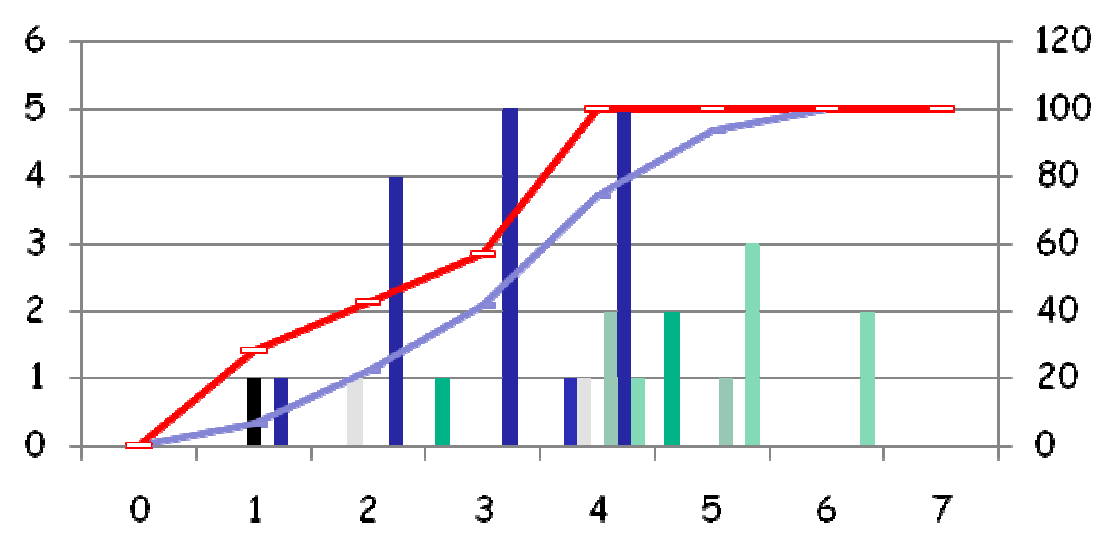




\section{SAGE Summary}

- SAGE is so effective at finding bugs that, for the first time, we face "bug triage" issues with dynamic test generation

- What makes it so effective?

- Works on large applications (not unit test)

- Can detect bugs due to problems across components

- Fully automated (focus on file fuzzing)

- Easy to deploy (x86 analysis - any language or build process !)

- Now, used daily in various groups inside Microsoft 


\section{More On the Research Behind SAGE}

- Challenges:

- How to recover from imprecision in symbolic execution? PLDI'05

- How to scale symbolic exec. to billions of instructions? NDSS'08

- How to check efficiently many properties together? EMSOFT'08

- How to leverage gram. specs for complex input formats? PLDI'08

- How to deal with path explosion in large prgms? POPL'07, TACAS'08

- How to reason precisely about pointers? ISSTA'09

+ research on constraint solvers (Z3, disolver, ....) 


\section{Extension: Active Property Checking}

- Traditional property checkers are "passive"

- Purify, Valgrind, AppVerifier, TruScan, etc.

- Check only the current concrete execution

- Can check many properties at once

- Combine with symbolic execution "active"

- Reason about all inputs on same path

- Apply heavier constraint solving/proving

- "Actively" look for input violating property

- Ex: array ref $a[i]$ where $i$ depends on input, $a$ is of size $c$

- Try to force buffer over/underflow: add " $(i<0)$ OR ( $i>c)$ " to the path constraint; if SAT, next test should hit a bug!

- Challenge: inject/manage all such constraints efficiently... 


\section{Ext.: Grammar-Based Whitebox Fuzzing}

- Input precondition specified as a context-free grammar

- Avoids path explosion in lexer and parser

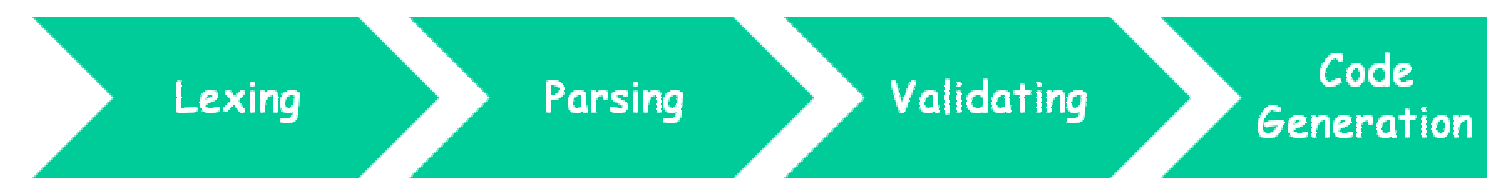

- Faster, better and deeper coverage for applications with structured inputs (XML, etc.)

\begin{tabular}{|l|c|c|c|}
\hline $\begin{array}{l}\text { generation strategy } \\
\text { (each ran 2 hours) }\end{array}$ & \#inputs & $\begin{array}{c}\text { total } \\
\text { coverage }\end{array}$ & $\begin{array}{c}\text { coverage in } \\
\text { code gen }\end{array}$ \\
\hline blackbox fuzzing & 8658 & $14 \%$ & $51 \%$ \\
\hline whitebox fuzzing & 6883 & $15 \%$ & $54 \%$ \\
\hline grammar-based blackbox fuzzing & 7837 & $12 \%$ & $61 \%$ \\
\hline grammar-based whitebox fuzzing & 2378 & $20 \%$ & $82 \%$ \\
\hline
\end{tabular}




\section{Ext.: Compositionality $=$ Key to Scalability}

- Problem: executing all feasible paths does not scale!

- Idea: compositional dynamic test generation

- use summaries of individual functions (arbitrary program blocks) like in interprocedural static analysis

- If $f$ calls $g$, test $g$ separately, summarize the results, and use $g$ 's summary when testing $f$

- A summary $\varphi(g)$ is a disjunction of path constraints expressed in terms of input preconditions and output postconditions:

$$
\varphi(g)=\vee \varphi(w) \quad \text { with } \quad \varphi(w)=\operatorname{pre}(w) \wedge \operatorname{post}(w)
$$

expressed in terms of $g$ 's inputs and outputs

- g's outputs are treated as symbolic inputs to a calling function $f$

- Can provide same path coverage exponentially faster! 


\section{Conclusion: Blackbox vs. Whitebox Fuzzing}

- Different cost/precision tradeoffs

- Blackbox is lightweight, easy and fast, but poor coverage

- Whitebox is smarter, but complex and slower

- Note: other recent "semi-whitebox" approaches

- Less smart (no symbolic exec, constr. solving) but more lightweight: Flayer (taint-flow, may generate false alarms), Bunny-the-fuzzer (taint-flow, source-based, fuzz heuristics from input usage), etc.

- Which is more effective at finding bugs? It depends...

- Many apps are so buggy, any form of fuzzing find bugs in those!

- Once low-hanging bugs are gone, fuzzing must become smarter: use whitebox and/or user-provided guidance (grammars, etc.)

- Bottom-line: in practice, use both! (We do at Microsoft) 


\section{Future Work (The Big Picture)}

- During the last decade, code inspection for standard programming errors has largely been automated with static code analysis

- Next: automate testing (as much as possible)

- Thanks to advances in program analysis, efficient constraint solvers and powerful computers

- Whitebox testing: automatic code-based test generation

- Like static analysis: automatic, scalable, checks many properties

- Today, we can exhaustively test small applications, or partially test large applications

- Biggest impact so far: whitebox fuzzing for (Windows) security testing

- Improved security for a billion computers worldwide!

- Next: towards exhaustive testing of large application (verification)

- How far can we go? 